\title{
Biosensorprinzipien für die patientennahe Labordiagnostik (POCT)
}

\author{
Peter B. Luppa
}

Institut für Klinische Chemie und Pathobiochemie, Klinikum rechts der Isar der TU München, Ismaninger Str. 22, 81675 München

\section{Zusammenfassung}

Die Präsentation definiert die analytischen Prinzipien für die patientennahe Labordiagnostik (Point-of-care testing, POCT) und kategorisiert die verschiedenen Analysegeräte, die derzeit im klinischen Alltag eingesetzt werden. Die folgenden Kategorien werden besprochen:

Typ 1 Qualitative Methoden

Typ $2 »$ Unit-use«-Systeme

Typ 3 Benchtop-Instrumente

Typ 4 Viskoelastische Gerinnungsanalysatoren

Typ 5 Kontinuierliche Messmethoden

Typ 6 Molekularbiologie-basierte Analysatoren

Einzelne Beispiele zeigen den derzeitigen Technologiestand für Anwendungen im stationären und ambulanten Bereich sowie für die Patientenselbsttestung auf.

Besonderer Fokus wird auf die Biosensor-Transducertechnologien bei elektrochemischen und optischen Analysesystemen gelegt. Diese ermöglichen quantitative Bestimmungen von Einzelanalyten, aber auch Multiplex-Testungen in menschlichen Untersuchungsmaterialen wie Blut, Urin oder Liquor cerebrospinalis.

\section{Allgemeine analytische Prinzipien}

Die POCT-Technologie hat - ausgehend von einfachen Handgeräten für die Blutglukosebestimmung und ersten Blutgasanalysatoren in den 1960er Jahren - die unterschiedlichsten Methodiken und Anwendungen hervorgebracht. Die allgemeinen Prinzipien [1] werden nachfolgend kurz erläutert. Eine hilfreiche erste Übersicht gibt dabei der Aufbau dieser Analysesysteme:

- $\quad$ Detektoreinheit,

- $\quad$ Transducer-Einheit,

- $\quad$ Probenaufgabe/Fluidikeinheit,

- $\quad$ Processing-Einheit.

POCT-Systeme entwickelten sich aber auch aus den sog. Schnelltests (z. B. Schwangerschaftstest). Bei diesen einfachen Systemen entspricht das menschliche Auge der Signalauslesung im Transducer. Alle weiteren komplexeren Analysesysteme werden dann als Sensor betrachtet, wenn durch den Transducer das Signal ausgelesen und in ein elektrisches Signal umgewandelt wird. 


\section{Detektor}

Der Detektor ist als selektiver Signalgeber anzusprechen. Dies ist dann der Fall, wenn auf einer Oberfläche des Detektor/Transducer-Systems eine spezifische Erkennungsschicht immobilisiert wurde. Diese Erkennungsschicht kann sowohl biospezifische als auch chemospezifische Eigenschaften aufweisen. Die Oberfläche kann aus unterschiedlichen Kunststoffmaterialien, Glas, Silizium oder Edelmetallen bestehen. Metalloberflächen können mit oder ohne funktionalisierte Zwischenschicht hergestellt werden.

Die Signalgenerierung kann auf verschiedene Weise mit oder ohne Substrataddition realisiert werden. Zum einen gibt es optische Oberflächenanalytikmethoden wie z. B. die Ellipsometrie oder die Oberflächenplasmonresonanz, die von der Oberfläche spezifische Informationen generieren. Es können zum anderen aber auch spektroskopische Methoden eingesetzt werden wie Absorptions-, Fluoreszenzoder (Chemi-)Lumineszenzmessungen. Weiterhin werden elektrochemische Methoden wie Amperometrie und Potenziometrie oder sogar optische Bewegungsanalysen zur Signalgenerierung eingesetzt. In der Tabelle wird die Signalgenerierung nochmals zusammengefasst.

\begin{tabular}{|c|c|c|}
\hline Signalgenerierung & Oberfläche & Erkennungsschicht \\
\hline $\begin{array}{l}\text { Markierungsfreie optische } \\
\text { Oberflächenanalytik: } \\
\text { Mikroreflektometrie } \\
\text { - Ellipsometrie } \\
\text { - Reflektometrische } \\
\text { Interferenzspektroskopie } \\
\text { Mikrorefraktometrie } \\
\text { - Oberflächenplasmonresonanz } \\
\text { - Gitterkoppler } \\
\text { - Mach-Zehnder Interferometrie }\end{array}$ & 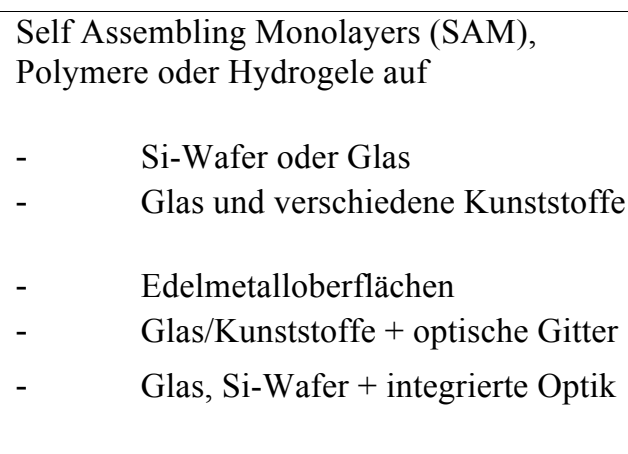 & $\begin{array}{l}\text { Biospezifisch: } \\
\text { Antikörper } \\
\text { Antigene } \\
\text { Rezeptoren } \\
\text { Liganden } \\
\text { andere Bindungsproteine } \\
\text { Enzyme (Substratumsatz, } \\
\text { Enzymaktivierung oder - } \\
\text { inhibition) } \\
\text { Aptamere } \\
\text { DNA/RNA }\end{array}$ \\
\hline $\begin{array}{l}\text { Spektroskopie: } \\
\text { Fluoreszenz, (Chemi-) } \\
\text { Lumineszenz, Totale Interne } \\
\text { Reflektionsfluoreszenz }\end{array}$ & $\begin{array}{l}\text { - Glas oder Kunststoffmaterialien } \\
\text { (Mikrotiterplatten, Mikroskopslides), bei } \\
\text { TIRF + integrierter Optik oder optischen } \\
\text { Gittern } \\
-\quad \text { Si-Wafer }\end{array}$ & $\begin{array}{l}\text { (Protein-)Scaffolds } \\
\text { lebende Zellen } \\
\text { Chemospezifisch: } \\
\text { »molecular imprints« } \\
\text { Indikatoren }\end{array}$ \\
\hline $\begin{array}{l}\text { Reflexion } \\
\text { Absorption } \\
\text { Trübungsmessung } \\
\text { Optische Bewegungsanalyse }\end{array}$ & Homogene Phase & \\
\hline $\begin{array}{ll}\text { Elektrochemie: } \\
- & \text { Amperometrie } \\
- & \text { Potenziometrie } \\
- & \text { Konduktivität } \\
- & \text { Coulometrie }\end{array}$ & Edelmetallelektroden & Ionenselektive Membranen \\
\hline
\end{tabular}

\section{Transducer}

Der Transducer entspricht einem Messwandler, der optische, elektrochemische oder andere Auslesungen (thermometrisch, mikrogravimetrisch, magnetisch etc.) ermöglicht. Der Transducer wandelt die biologische/chemische Reaktion in eine physikalische Größe, die in ein Signal bzw. in digitale Daten 
umgewandelt wird und danach weiterverarbeitet werden kann. Das Signalverarbeitungssystem konditioniert dabei das Sensorsignal durch elektronisches Glätten und Rauschfilterung.

Besonders bemerkenswert ist wie oben bereits erwähnt die biospezifische Erkennungsschicht auf dem Detektor/Transducer-System [2]. Dabei handelt es sich häufig um immobilisierte Antikörper oder um Enzyme. Während die Antikörper durch Assoziation mit dem Analyten als biospezifische Erkennungsschicht fungieren, wird bei den Enzymen meistens die katalytische Reaktion nach Zusatz von Substrat erfasst. Als Beispiel sind hier die Glukosesensoren zu nennen. Bindungsproteine können als »recognition proteins«, z. B. bei einer Blutgerinnungsanalyse, eingesetzt werden [3].

Unter chemospezifischen Erkennungsschichten versteht man dagegen alle Arten von ionen- und molekülselektiven Membranen, molekulare Abdrücke (»imprints«) oder Indikatormoleküle, die durch Selektivität gegenüber dem Analyten als Erkennungsschicht geeignet sind.

\section{Probenaufgabe/Fluidikeinheit}

Die Probenaufgabe bei POCT-Geräten (zumeist von kapillär gewonnenem Vollblut) kann im Gegensatz zur oft komplexen Einschleusung einer Patientenprobe in klinisch-chemische Analysengeräte stark vereinfacht ablaufen. Die POCT-Geräte nutzen dazu häufig eine Fluidikeinheit, die zumeist aus Einwegkomponenten aufgebaut ist. Das Fluidikformat kann wie folgt definiert werden:

- $\quad$ Systeme mit absorbierenden Materialien, Durchflusszelle, lateralem Flow oder (Immuno-) Chromatographie,

- $\quad$ Kassetten-, Kapillar-, Schlauch- oder Zentrifugalsysteme.

Dabei wird die Fließbewegung durch Vakuum, Pumpen oder Zentrifugalkräfte aufrechterhalten; es werden alternativ aber auch die absorptiven Eigenschaften vieler Materialien, die Kapillarkraft sowie die Elektroosmose ausgenutzt [3]. Viele Fluidiksysteme bestehen aus Kassetten, die sämtliche Reagenzien in Segmenten bereitstellen und durch eine Öffnung mit Probenmaterial beschickt werden.

Einfache »Unit-use«-POCT-Geräte (wie z. B. Blutglukosemessgeräte) benutzen oft Teststreifen, die für eine Messung manuell in das Gerät eingesetzt werden. Danach wird das Probenmaterial ebenfalls manuell an die Auftragszone gebracht und selbsttätig durch Kapillarkräfte in den Streifen eingesaugt.

Blutgasanalysatoren dagegen saugen eine Blutprobe zumeist direkt aus der Spritze oder Kapillare mit Hilfe von Rollerpumpen und Schlauchsystemen an.

\section{Processing-Einheit}

Zur Weiterverarbeitung der vom Transducer aufbereiteten Messsignale benutzen viele POCT-Geräte gängige Computerbetriebssysteme. Damit werden nicht nur die (quantitativen) Resultate sichtbar gemacht (Display, Drucker etc.), sondern es werden auch sämtliche analysebegleitenden Berechungen (Kalibrierung etc.) und das Datenmanagement (Speicherung von Qualitätskontrollmessungen, Patientenergebnissen etc.) im Gerät durchgeführt. Schnittstellen zu POCT-Verarbeitungsprogrammen im Rahmen einer POCT-Koordinationsstelle in einem Krankenhaus-Netzwerk (Hospital Information System) sind meist vorhanden. 


\section{Gerätekategorien}

Auf dem In-vitro-Diagnostika-Markt finden sich die unterschiedlichsten Geräte, von den kompakten »handhelds « bis zu den Tischgeräten, die komplizierte Analysensysteme für Küvettentests oder trägergebundene Verfahren darstellen. Das Methodenspektrum reicht von einfachen Teststreifen bis hin zu komplexen immunchemischen Analysen. Die POCT-Geräte sind in der Regel nahezu vollständig automatisiert und erfordern von der Probenvorbereitung bis zum Testergebnis nur einfachste Handgriffe des Benutzers.

In den letzten Jahren haben sich folgende Instrumentenkonzepte durchgesetzt:

- $\quad$ Handgeräte, z. B. Glukosemessgeräte (entspricht einem »Unit-use«-System; s. unten),

- $\quad$ andere »Unit-use«- oder »Multi-use«-Kassettensysteme,

- $\quad$ stationäre Tischgeräte, z. B. Blutgas-Elektrolyt-Substrat-Analysatoren,

- $\quad$ \Lab-on-a-chip«-Systeme.

Die POCT-Analytik ist - verglichen mit der Analytik im Zentrallabor - prinzipiell mit erheblich höheren Kosten verbunden. Dies ist darauf zurückzuführen, dass die speziell vorkonfektionierten Reagenzien kostenintensiv sind. Zudem sind die Vorhaltungskosten (Kapitalbindung durch Beschaffung und Wartung vieler einzelner Analysesysteme, Reagenzienbevorratung etc.) hoch. Dagegen spielen für das Krankenhaus als übergeordnete wirtschaftliche Einheit mögliche Einsparungen durch die Verlagerung von Analytik vom Zentrallabor zu den dezentralen POCT-Plätzen kaum eine Rolle, da dieser Transfer zumeist keinen Wegfall des entsprechenden Arbeitsplatzes im Zentrallabor zulässt.

Im Folgenden werden die einzelnen Instrumentenkonzepte vorgestellt. Einzig die »Lab-on-a-chip«Systeme sollen an dieser Stelle nicht weiter besprochen werden, da die Entwicklung derartiger Chips zurzeit zwar sprunghaft voranschreitet, die Anwendungen in der Medizin jedoch noch nicht ausreichend evaluiert sind.

\section{Typ 1 - Qualitative POCT-Methoden}

Diese qualitativen Teste diskriminieren zwischen plus/minus-Resultaten und sind zumeist als Teststrips anzusprechen. Das Messsignal kann entweder direkt visuell abgelesen werden oder wird mittels eines einfachen Auslesegeräts erfasst. Die Detektionprinzipien reichen von chemischen Indikatorreaktionen bis zu immunologischen Antigen/Antikörper-Interaktionen (z. B. Lateral Flow Assays).

Die Strips bestehen aus einem festen Trägermaterial und einer porösen Matrix, die mit lyophilisierten Reagenzien versetzt ist. Die Patientenprobe (Urin, Blut, Stuhl, Liquor, Abstrichmaterial etc.) wird auf den Stip aufgebracht und startet die analytische Reaktion durch das Eindringen in die Strip-Schicht.

\section{Typ 2 - »Unit-use«-Systeme}

»Unit-use«-POCT-Geräte stellen die einfachste Form eines quantitativen POCT-Gerätes dar. Die eigentliche analytische Detektorreaktion läuft auf dem Teststrip ab, das Auslesegerät generiert nur den ablesbaren Messwert. Unit-use Geräte haben i. A. folgende Charakteristika:

- $\quad$ Für jede Messung werden einzeln verpackte Reagenzien/Strips benutzt. »Unit-use«-Reagenzien sind für Einzelbestimmungen portioniert und mit einer Untersuchung verbraucht.

- $\quad$ Sie benutzen zur Testung unprozessiertes Vollblut, wobei das Testmenü sehr unterschiedlich ist und von den verwendeten Sensoren abhängt. 
- $\quad$ Charakteristisch ist, dass die Sensoren in den Test-Strip und nicht in das Gerät eingearbeitet sind. Häufig werden dabei trockenchemische Verfahren benutzt, z. B. glukoseumsetzende Enzyme, die auf Reagenzienstrips immobilisiert sind. Bei diesen Geräten wird eine Kalibrierung zumeist durch elektronische oder physikalische Standards ersetzt.

- $\quad$ Komplexere Kassettengeräte verfügen dagegen über automatische Kalibrierungen, die in festgelegten Zeitabständen ablaufen.

- $\quad$ Es gibt Geräte, die nur für eine einzige Kenngröße ausgelegt sind. Häufig können jedoch Gerätesysteme über den Einsatz unterschiedlicher Strips verschiedene Kenngrößen messen.

Die angewandten Technologien für »Unit-use«-Geräte reichen von elektrochemischen Methoden (Blutglukosegeräte) über Immunosensoren (z. B. Cobas h 232 von Roche) bis zu Dünnfilmsensoren (z. B. i-STAT von Abbott). Die Analysezeiten betragen häufig nur wenige Sekunden bis eine Minute.

\section{Typ 3 - Benchtop-Instrumente}

Im Allgemeinen sind Benchtop-Geräte als komplexe Analysesysteme nicht direkt am Patientenbett lokalisiert, sondern in den Funktionsbereichen einer Intensivstation, in Notfallambulanzen oder in Arztpraxen.

Man unterscheidet bei den Benchtop-Geräten:

- $\quad$ klinisch-chemische, spektrophotometrische Vielkanalgeräte (z. B.: Abaxis Piccolo Xpress),

- $\quad$ klinisch-chemische Teststreifen/Kartuschengeräte (z. B.: Roche Reflotron),

- $\quad$ hämatologische Vielkanalgeräte (z. B. Sysmex pocH-100i),

- $\quad$ Blutgasgeräte mit/ohne Oxymetrie und Elektrolyt-/Substratmessungen (z. B. von IL, Keller Medical, Nova, Radiometer, Roche und Siemens),

- $\quad$ immunologische Vielkanalgeräte (z.B. Radiometer AQT90).

Die in den unterschiedlichen Systemen eingesetzte Technologie stammt weitestgehend von den im Zentrallabor benutzten mechanisierten Analysesystemen ab und wurde hinsichtlich

Bedienerfreundlichkeit, Miniaturisierung und Analysegeschwindigkeit (meist nur wenige Minuten) optimiert. Dagegen wurden die Blutgasanalysatoren bereits vor Jahrzehnten primär für die POCTAnalytik konzipiert und seither mit einem ständig steigenden Menüangebot komplettiert.

\section{Typ 4 - Viskoelastische Gerinnungsanalysatoren}

Die kombinierte Analyse der Plasmagerinnung, der Thrombozytenfunktion und der Fibrinolyse wird viskoelastische Gerinnungstestung genannt [4]. Viskoelastische Gerinnungsanalysatoren weisen einen hohen Grad an Komplexizität auf, sie sind daher nur bedingt POCT-tauglich. In der Regel werden sie von speziell geschultem Personal vor Ort (z. B. im Operationssaal) bedient, oder die Analyse wird vom Zentrallabor elektronisch in den klinischen Bereich in Echtzeit übertragen. Beispiele sind die Rotationsthrombelastographie mittels des ROTEM (TEM International) oder das Sonoclot Gerät von Sienco. Eine Analyse der Plättchenfunktion ist auch durch eine in vitro Blutungszeit-Messung oder durch eine optische Aggregometrie möglich. Beispiele sind das PFA 100 von Siemens oder das VerifyNow von Accumetrics.

\section{Typ 5 - Kontinuierliche POCT-Messmethoden}

Kontinuierliche Messmethoden sind vor allem für das Glucose-Monitoring entwickelt worden und zum Teil mittlerweile auch kommerziell verfügbar [5]. Mittels eines minimal-invasiven Mikrodialyse- 
Katheters im Subkutangewebe gelingt die kontinuierliche Messung bis zu $>72$ Stunden. Andere nichtinvasive Methoden wie die Mikroporation oder optische Techniken einer direkten transkutanen Messung von metabolischen Kenngrößen konnten sich in den letzten Jahren nicht durchsetzen. Dies liegt zum großen Teil damit zusammen, dass die menschliche Haut bzgl. Dicke, Pigmentation, Behaarung, aber auch bzgl. physiologischer Phänomene wie Feuchtigkeit oder Salzgehalt sehr uneinheitlich ist.

\section{Typ 6 - Molekularbiologie-basierte POCT-Analysatoren}

Es gibt derzeit vielfältige Bestrebungen molekularbiologische Techniken (zumeist basierend auf der Polymerase chain reaction, PCR) auch für POCT anwendbar zu machen. Derartige Ansätze sind jedoch technisch anspruchsvoll (Extraktion von DNA oder RNA aus z. B. Abstrichmaterial), sodass die Analysezeit sicherlich auch in Zukunft über den der sonstigen POCT-Methoden liegen wird. Alternativ könnten isothermale Amplifikationsmethoden zu kürzeren Analysezeiten führen. Aufgrund der Komplexität der Testdurchführung und der Resultatinterpretation werden zukünftige schnelle Nucleic Acid Testing (NAT)-Verfahren nur teilweise in klinischen Bereichen zu finden sein. Ähnlich wie bei Typ 5 werden derartige Geräte oft im Zentrallabor betrieben werden [6]. Dennoch wird der schnelle und quantifizierende Nachweis von DNA/RNA von bakteriellen und viralen Krankheitserregern den Klinikern wertvolle diagnostische Informationen liefern. Derzeit ist das GeneXpert System von Cepheid ein Vorreiter, was die Vollautomation und die Integration aller real-time PCR-basierter NAT-Schritte (Probenvorbereitung, DNA/RNA-Amplifikation und DNA/RNA-Detektion) anbelangt.

\section{Literatur}

1. Luppa PB, Müller C, Schlichtiger A, Schlebusch H. Point-of-care testing (POCT): Current techniques and future perspectives. Trends Anal Chem 2011;30:887-898.

2. Luppa PB, Sokoll LJ, Chan DW. Immunosensors - Principles and applications to clinical chemistry. Clin Chim Acta 2001;314:1-26.

3. Price CP, St John A, Hicks JM. Point-of-care testing: what, why, when, and where? In: Price CP, St John A, Hicks JM (eds) Point-of care testing, 2nd edn. AACC Press, Washington, pp 3-12, 2004.

4. Ganter MT, Hofer CK. Coagulation monitoring: current techniques and clinical use of viscoelastic point-of-care coagulation devices. Anesth Analg 2008;106:1366-1375.

5. Corstjens AM, Ligtenberg JJ, van der Horst IC, Spanjersberg R, Lind JS, Tulleken JE, et al. Accuracy and feasibility of point-of-care and continuous blood glucose analysis in critically ill ICU patients. Crit Care 2006;10:R135.

6. Seme K, Mocilnik T, Komlos KF, Doplihar A, Persing DH, Poljak M. GeneXpert enterovirus assay: one-year experience in a routine laboratory setting and evaluation on three proficiency panels. J Clin Microbiol 2008;46:1510-1513. 